\title{
Health-Related Shape Analysis of 3D Body Scanner Data
}

\author{
C. Lovato ${ }^{*^{1}}$, C. Milanese ${ }^{1}$, F. Piscitelli ${ }^{1}$, C. Zancanaro ${ }^{1}$, A.Giachetti ${ }^{2}$ \\ ${ }^{1}$ Dept. of Neurological, Neuropsychological, Morphological and Movement Sciences, Univ. of Verona \\ ${ }^{2}$ Dept. of Computer Science, University of Verona
}

\begin{abstract}
Body scanner technology is becoming widely available and provides plenty of information about body anatomical structure. Nevertheless, there isn't yet a well-established agreement about the correct way to turn the scanner digital data into knowledge about health related parameters.

In this paper, we compare easily detectable geometrical features obtained from 3D scans of female obese (BMI > 30) subjects with body composition (measured with a DXA device) of the same subjects, in order to investigate which measurements on shape descriptors better correlate with torso and body fat. The results obtained show that some of the tested geometrical parameters have a relevant correlation, while other ones do not strongly correlate with body fat. These results support the role of digital anthropometry in investigating health-related physical characteristics and encourage the realization of further studies analyzing the relationships between shape descriptors and body composition.
\end{abstract}

Keywords: 3d body scanning, health-related anthropometrics, body fat, automatic body segmentation, body composition

\section{Introduction}

The increased availability of whole body scanner devices provides an efficient way to acquire automatically anthropometric parameters that can be, for example, used in medical studies. Body shape measurements (e.g. W/H ratio) can be in fact used as an indirect assessment of fat content and distribution and are known to correlate with the risks of developing certain diseases, e.g. the metabolic syndrome and diabetes. 3D scanners are able to provide complete digital models of the subjects bodies, encoding a huge amount of information that can be processed automatically making it easier for physicians to evaluate compact descriptors that can be correlated with body composition or diagnostic variables.

From the digital models, in fact, it is possible to extract not only a wide set of measurements usually picked up from manual anthropometry, but also, using geometry processing techniques, to derive parameters related to curvatures, statistical variations of local and global properties, salient points location, etc. that may well capture most relevant shape variations in the analyzed population.

It may be difficult, however, to compare manual anthropometric measurements and parameters computed on digital models, because the first ones are based on the identification of anatomical landmarks, obtained through observation and palpation by expert anthropometrists which are difficult to be located only on the basis of marker-less digital acquisitions.

For this reason we decided not to compare directly manually anthropometry with digital evaluation of geometrical parameters, but we focused our investigation on measurements that are objective and easily (or automatically) computed from optical scanner data to see if they correlates with composition data to a comparable (or better) extent of traditional measurements.

We developed an automatic tool, device independent and tolerant to variable changes in pose, able to process raw body scanner data performing re-meshing and body segmentation, and skeletonization and providing automatically a set of geometrical descriptors of the torso region. We computed this set of parameters on 3D models of obese female subjects that also underwent traditional anthropometric procedures and dual-energy X-ray absorptiometry (DXA).

To investigate the medical interest of shape related parameters, we evaluated the ability of the computed descriptors and of a set of traditional anthropometric measurements (e.g. selected diameters and lengths), to predict total body fat or trunk fat (as measured by DXA) by means of linear regression analysis. The use of 3D body scanners for studying obesity is not entirely new; several scanner manufacturers provide software tools to collect sets of measurements that can be used to analyze body shape, and these anthropometric data have been recently correlated with metabolic parameters in validation studies [1,2,3]. A relevant drawback of these tools is that they are not standardized: parameters often are strongly dependent on the acquisition device, and on pose [4], and there are not advanced tool to extract all the relevant medical information from the whole geometry.

* email: chr_lovato@yahoo.it 
A company named Select Research UK recently launched a system called BVI, Body Volume Index [5] that should provide a highly accurate electronic measurement for obesity and is designed to be a potential replacement to the Body Mass Index; the system is currently being experimented. This is, however, a closed solution requiring dedicated hardware and details have not been disclosed.

The novelty of our approach is that we aim at working out a new measurement procedure using parameters that could be easily obtained from on any sufficiently accurate 3D model, possibly with few pose requirements. The software tool and the experimental study here described are designed as first steps in the development of an open system for the analysis of body shape features related to obesity and other diseases.

\section{Material and methods}

We analyzed data from 25 obese otherwise healthy female subjects, aged 20-60 years and BMI 30-40 $\mathrm{kg} / \mathrm{m}^{2}$. The subjects underwent DXA scanning, optical 3D scanning and standard anthropometric procedures [6]. During scanning and anthropometry, subjects wore close-fitting underwear. During DXA examination subjects wore light garment, transparent to X-rays.

For every single subject, 3D scanning, DXA and manual anthropometry were performed sequentially in one session.

\subsection{DXA scanner}

Dual-Energy X-rays absorptiometry represents the de-facto standard measuring tool for body composition analysis. It allows to assess body composition in terms of fat, lean and mineral mass at the total body as well as regional level [7]. The DXA device used in this experiment is the DXA QDR Explorer W (Hologic, MA, USA) of the faculty of Motor Sciences of Verona University. The body composition assessment consists of an acquisition step and a subsequent definition of body regions on the 2D acquisition performed by an operator (Fig. 1).

Main results of DXA analysis consists in whole-body composition parameters and regional composition data. For our experiments we chose three parameters:

1. Trunk Fat

2. Subtotal Fat

3. Subtotal Fat Percentage

Trunk Fat is the total fat detected in the trunk region (as defined by the operator according to stringent anatomical landmarks on the 2D acquisition); Subtotal Fat and Subtotal Fat percentage refer to the fat of the whole body without the head; the reason for that is large amount of mineralized tissue in the skull, preventing reliable evaluation of the head fat component. Those last two parameters are well-known risk factors for metabolic alterations and disease.

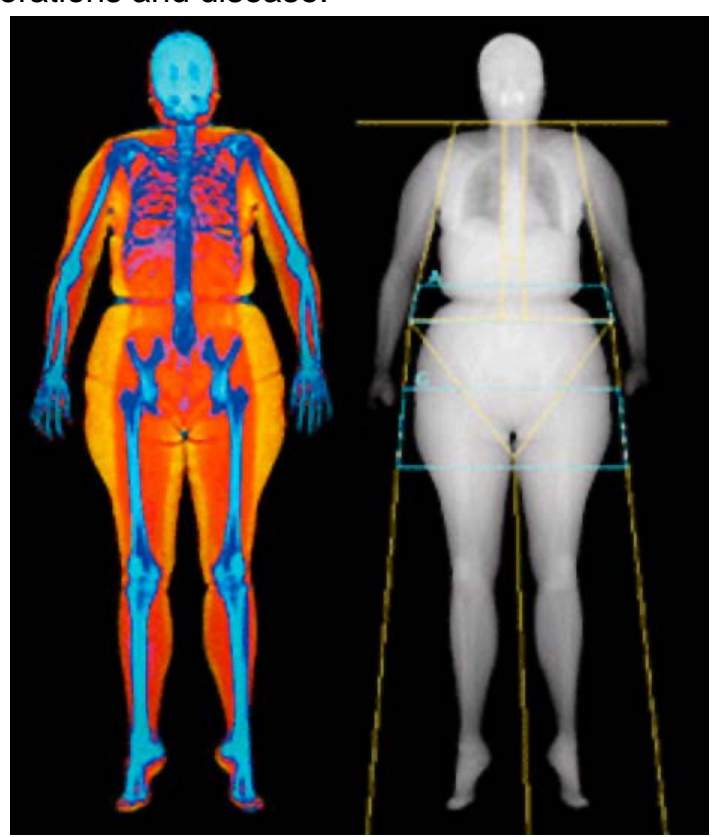

Fig. 1. Whole body composition (BC) images output of a DXA exam. On the left: BC in false colors. On the right: regions setting by means of template defined by colored lines (source Hologic) 


\subsection{D body scanner}

3D surface model of subjects was acquired by means of a Breuckmann BodySCAN, that uses high-speed cameras and a proprietary algorithm to detect the actual position of body surface by means of projected diffraction fringes. Breuckmann bodySCAN typical model consists of about 400.000 points with a resolution ranging from $0.2 \mathrm{~mm}$ to $1.4 \mathrm{~mm}$, and an acquisition time of 2.5 or 5.5 seconds, depending on the selected mode.

The scanner output data consists of a triangular mesh obtained by the registration and merging of the points acquired by the different cameras. Resulting meshes are, however, not very clean, presenting various types of defects like holes, non-manifold edges, bad shaped triangles and outliers, so a pre-processing step is mandatory.

Meshes nodes include also gray-scale information. This is extremely important in order, for example, to acquire landmarks position useful for automatic or computer assisted measurements by exploiting natural or added texture (i.e. skin markers).

\subsection{Human analyzer tool}

Automatic computation of geometrical parameters from generic body scanner data was performed with a specifically designed software tool, able to load 3D meshes and provide directly geometrical parameters without the necessity of user intervention.

It performs a preprocessing step able to remove spurious parts of the model and creating a new watertight mesh with the Poisson algorithm. This procedure is based on VCG/Meshlab scripts [7], integrated in the software package, implemented for the other parts with custom C++ code.

The subsequent segmentation procedure has been derived from the method described in [8], that is based on a curve skeleton extraction and its partitioning based on the extraction of features on the major skeletal leaves. In this way it is possible to detect and recognize points where head and limbs join the body trunk. This procedure is however not precise when the captured shape is not optimal, e.g. arms are partially attached to the trunk or legs are in contact under the crotch; these problems are often encountered when dealing with obese subjects.

For this reason we modified it by using special heuristics to refine the position of the feature points used to define the body segmentation, e.g. attachments of limbs to the trunk.

First of all, we observed that, where legs and arms are attached to the trunk, their direction should be approximately parallel to the trunk axis. This means that we can use the detected endpoints of the limbs to define a plane approximately including the trunk axis, so we can define an approximate trunk axis, and approximate transverse and antero-posterior directions.

We then move the detected leg centerline endpoints toward the trunk until the antero-posterior distance along the line joining the leg centerline endpoints is not lower than at its extrema.

We adjust then the crotch position and the trunk axis direction analyzing the trunk sections perpendicular to the previously detected axis near detected endpoints.

Lateral trunk limits (transverse distance from the axis) are obtained detecting the lower part of the arms branching and symmetrizing the result, finally the shoulder position is searched as the upper points of the intersection of the planes defining these limits with the trunk geometry.

Fig. 2 shows an example of automatic body segmentation result with the curve skeleton, its initial partitioning, the refined limb extrema defining a reasonable stick figure even for overweight subjects.

Known the body segmentation, the trunk centerline, transverse and antero-posterior directions, we can compute a number of parameters (lengths, girths, volumes, etc.), with accuracy that is obviously depending on acquisition protocols, body position, etc. In this work we selected a few parameters that we assumed could be reasonably accurate correlated with the body fat.

The software tool performs the automatic evaluation of the parameters (saved as a text file) as well as a segmented 3D surface, the computed curve skeleton and stick figure.

Most trunk measurements are made by means of analysis of profiles curves along trunk centerline, so that every profile point characterizes a trunk slice.

Typical profiles measures are antero-posterior diameter, latero-lateral diameter, mean diameter, eccentricity and curve length (the equivalent of "Circumference" in anthropometry) of the slice (Fig. 3).

In this way is simple to derive meaningful geometrical measures, like maximum antero-posterior diameter or maximum curve length. 

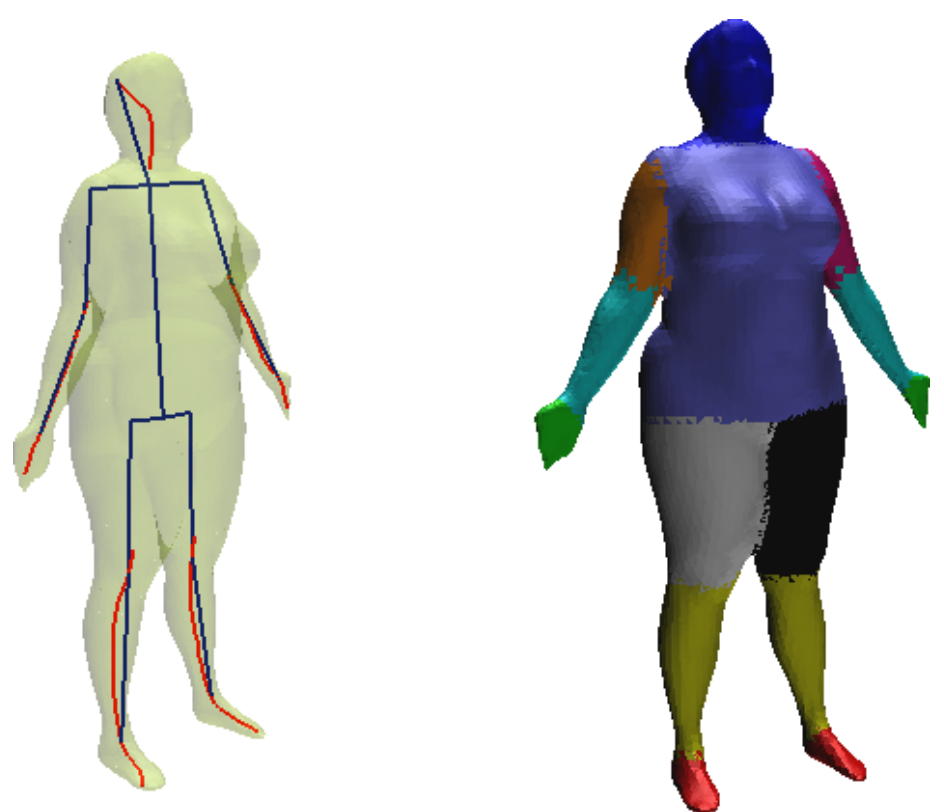

Fig. 2. Left: segmentation based simply in the extrema of tubular parts (defined by red centerline paths) fails in capturing the real position of limb endpoints due to the attachments of limb to the trunk. With the simple heuristics describe in the text our software can locate the correct position and obtain a reasonable stick figure (blue), body part segmentation (right) and the estimate of the axial, antero-posterior and transverse directions useful to evaluate the parameters considered in our study.

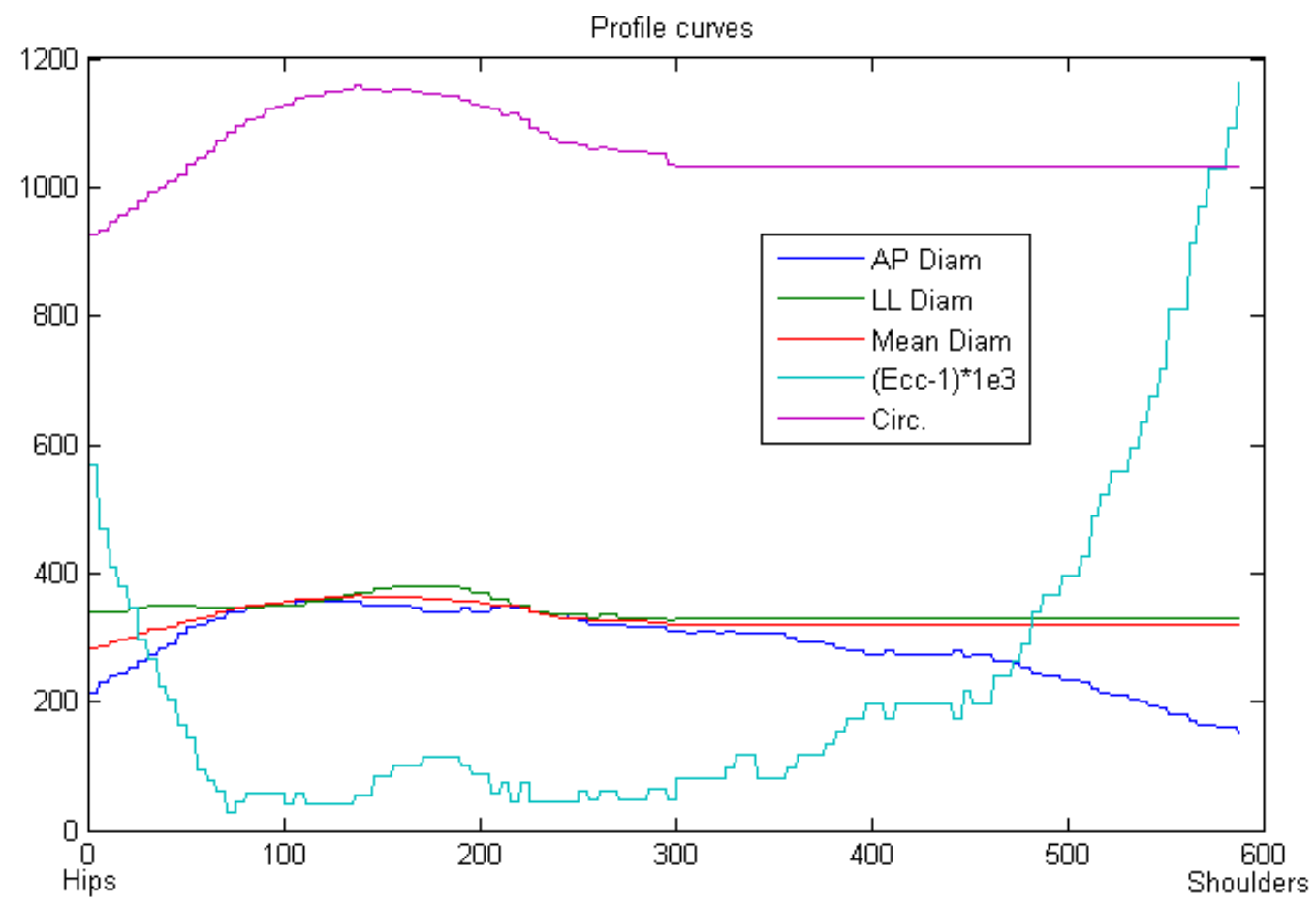

Fig. 3. Example of computed profile curves on the trunk. The curves represents antero-posterior, latero-lateral and mean diameters, eccentricity and slice circumference Eccentricity (maximum and minimum diameters ratio) profile has been rescaled for clarity. 


\subsection{Comparison}

We performed the comparison analysis of DXA data with automatic and manual measurements using Pearson's linear correlation coefficient.

Chosen DXA measurements were trunk fat, subtotal body fat and percentage of the former over subtotal body mass. Body composition parameters have been correlated with some standard anthropometric indexes and circumferences. Standard indices are usually employed to evaluate the health status of individuals. Some of them, like BMI and body mass are known to be correlated with fat parameters, because fat mass directly influence them.

We compared then the same DXA parameters with the digital measurements performed on 3D mesh summarized in Table 1.

We conceived the set of measurements to capture in various ways the trunk geometry. Some of them are similar to the standard anthropometric ones; some others are instead almost impractical with standard anthropometry.

We also focused on measurements that are reasonably not affected by inaccuracies in mesh acquisition and processing, as it would happen if they are performed in regions where the original mesh presents holes.

Table 1. Description of performed automatic digital measurements

\begin{tabular}{|l|l|}
\hline \multicolumn{1}{|c|}{ Name } & \multicolumn{1}{c|}{ Description } \\
\hline AP max & Maximum anteror-posterior diameter \\
\hline MD max & Maximum mean diameter \\
\hline Area max & Maximum of slice area \\
\hline Height/MD ratio & $\begin{array}{l}\text { Ratio between the height of the trunk (hips-shoulders height) and the } \\
\text { maximum mean diameter. This measurement gives a gross shape } \\
\text { description of the trunk proportions }\end{array}$ \\
\hline Trunk vol. & Volume of the whole trunk, computed as sum of the slice areas \\
\hline Abdomen subvol. & Volume of the lower part of the abdomen, from hips to height of max AP \\
\hline Min/max circ ratio & Ratio between minimum and maximum curve lengths \\
\hline Min/max area ratio & Ratio between minimum and maximum slice areas \\
\hline AP curv. & Curvature of the AP curve in the neighborhood the maximum value \\
\hline MD curv. & Curvature of the MD curve in the neighborhood the maximum value \\
\hline Circ. curv. & $\begin{array}{l}\text { Curvature of the Circumference curve in the neighborhood the maximum } \\
\text { value }\end{array}$ \\
\hline
\end{tabular}

\section{Results and discussion}

We used our custom software tool to extract the measurements listed in Table 1 on the digital models captured by the 3D scanner. Computation time is less than on minute per model on a Dell XPS17 laptop with an Intel Core 17 Q740 CPU and 8Gbyte of RAM, running Ubuntu Linux.

The resulting measurement reports were then imported in Matlab, together with DXA data and manual anthropometry measurements in order to perform statistical analysis.

In Table 2 Pearson's Correlation coefficients between three DXA body composition parameters and the anthropometric (standard and digital) measurements are shown. 
Table 2. Correlationm matrix of anthropometric measurements and DXA trunk fat, subtotal body fat, and \% subtotal body fat

\begin{tabular}{|l|c|c|c|}
\hline $\begin{array}{l}\text { Anthropo- } \\
\text { metric. Item }\end{array}$ & Trunk Fat & Subtotal Fat & $\begin{array}{c}\text { \% Subtotal } \\
\text { Fat }\end{array}$ \\
\hline \hline BMI & 0.8379 & 0.8466 & 0.4698 \\
\hline Weight & 0.8314 & 0.8693 & 0.3542 \\
\hline Hips C. & 0.7757 & 0.8970 & 0.6188 \\
\hline Waist C. & 0.7573 & 0.4793 & -0.0208 \\
\hline W/H ratio & 0.1587 & -0.2259 & -0.5192 \\
\hline AP max & 0.9128 & 0.7616 & 0.3877 \\
\hline MD max & 0.9316 & 0.8894 & 0.5224 \\
\hline Area max & 0.9367 & 0.8794 & 0.4838 \\
\hline Height/MD ratio & 0.5995 & 0.6235 & 0.5326 \\
\hline Trunk Vol * & 0.6196 & 0.2025 & 0.0495 \\
\hline \hline Abdomen subvol & 0.0863 & 0.3813 & 0.4183 \\
\hline Min/max circ ratio & 0.3221 & -0.0272 & -0.1023 \\
\hline Min/max area ratio & -0.2865 & -0.0325 & 0.0813 \\
\hline AP curv & -0.3148 & -0.3559 & -0.3165 \\
\hline MD curv * & -0.3611 & -0.0840 & 0.0704 \\
\hline Circ curv * & -0.3110 & -0.1948 & 0.0451 \\
\hline * Some outliers removed (see text) & & \\
\hline
\end{tabular}

* Some outliers removed (see text)

In three cases we took out from statistics one or two outliers (one from Trunk vol. and two from the curvatures). The table is subdivided in three groups of rows. In the first group, correlations with standard anthropometric measures are shown. The second group presents digital measurements that are strongly correlated with DXA measured fat. The third group shows digital measurements that are poorly or not correlated with DXA data.

In figures 4 and 5 the scatter plots for the firs and the second groups of measurements are shown.

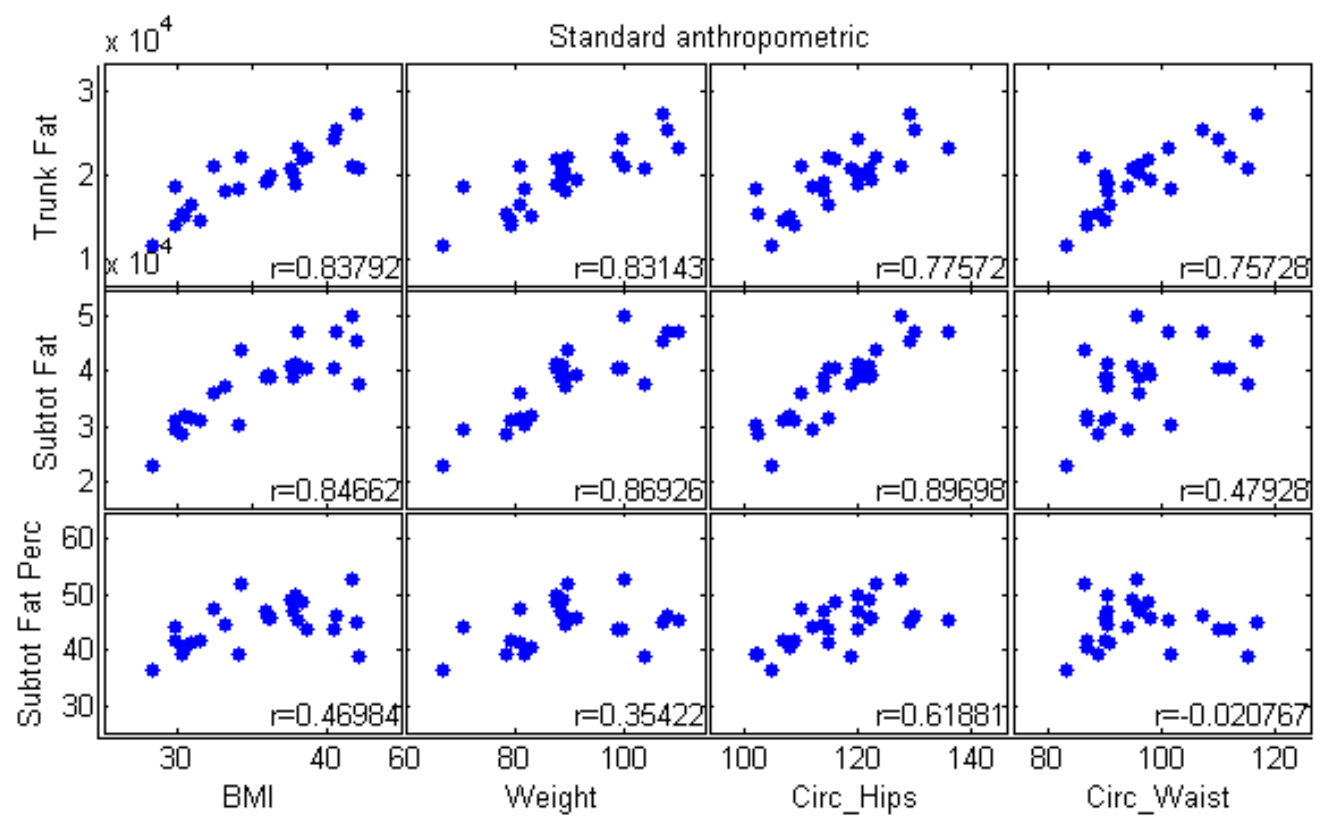

Fig. 4. Scatter plots of standard anthropometric measurements vs. DXA measurements. Pearson's Correlation Coefficients are indicated 


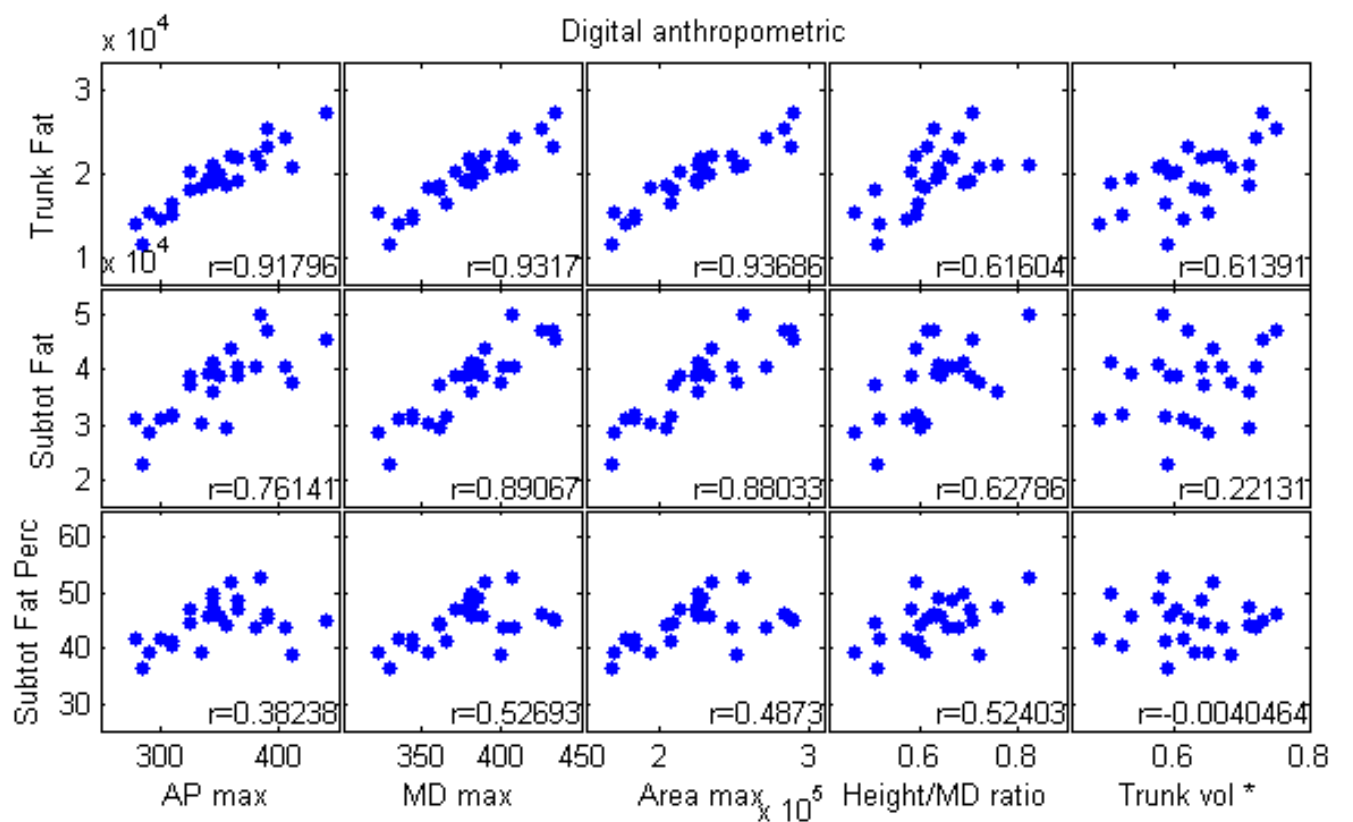

Fig.5. Scatter plots of selected digital measurements provided by the Human Analyzer software vs. DXA measurements. Pearson's Correlation Coefficients are indicated

Looking at results of standard measurements, we can see that BMI and body weight are well correlated with trunk and subtotal fat, as expected. Also hip circumference presents a high correlation index with subtotal fat.

As to digital measurements, there is a very high correlation (>0.9) of AP max, MD max and Area max with Trunk Fat, while the correlation with Subtotal Fat is high, but not as high as with Trunk Fat. Height/MD ratio and Trunk Vol correlate less $(r \simeq 0.6)$ with fat measurements.

As to the Subtotal Fat Percentage, correlations with evaluated standard and digital measurements are almost weak. Best found correlated measurements are $r \simeq 0.6$ with Hips Circ. and $r \simeq 0.5$ with MD max, Area max and Height/MD ratio.

\section{Conclusions}

We evaluated the ability of completely automatic measurements performed on body scanner data to predict body fat in obese female subjects. The results obtained suggest that some of the tested measurements can predict subtotal body fat similarly to standard anthropometric measurements, while they seem to correlate even better than tape-based anthropometric parameters with trunk fat. The high correlation of the maxima of mean diameter and section area with subtotal fat is not trivial, and the fact that simple measures on the trunk region are correlated with a whole body parameter, not easily estimated by other means deserves further investigation.

An interesting outcome of our analysis is that automatically computed measurements seem to be more correlated with trunk fat than similar parameters measured with the tape-based method. This could be due to the difficulty to locate precisely the landmarks on obese subjects and perhaps the possibly large deformations on the soft tissue created by the tape and calipers during the measurement process. This means that, even if the automatic measurement system is limited by several factors due to acquisition protocols and processing algorithm, indexes evaluated from the automatic system may be more effective than those coming from manual measurements (and, clearly, they are computed faster and with no user intervention).

We plan, however, to improve the accuracy of the automatic system and perform several validation tests on them, trying to solve problems that are not well treated by our current methods. A source of errors that make some parameters to lose precision, for example, is the pose of the arms of the subjects, which make latero-lateral measurements difficult where the arm adhered to lateral trunk surface. Moreover, in obese subjects the shape of the abdomen region is dependent on the underwear worn during the acquisition. This can introduce some errors on measurements localized in this region. 


\section{References}

1. Wang J, Gallagher D, Thornton JC, Yu W, Horlick M, and Pi-Sunyer FX. Validation of a 3-dimensional photonic scanner for the measurement of body volumes, dimensions and percentage body fat. Am J Clin Nutr, pages 809-816, 2006

2. Wells JC, Cole TJ, Bruner D, and Treleaven P. Body shape in American and British adults: between-country and inter-ethnic comparisons. Int J Obes., pages 152-159

3. Lin JD, Chiou WK, Weng HF, Fang JT, and Liu TH. Application of three-dimensional body scanner: observation of prevalence of metabolic syndrome. Clin Nutr., pages 1313-23, 2004

4. Karia P. Simmons. Body measurement techniques: a comparison of three-dimensional scanning and physical anthropometric methods. Ph.D dissertation,North Carolina State University, 2001

5. http://www.bodyvolume.com/ (accessed on august 2011)

6. Norton, K.,Olds, T., (Eds), (2001): "Anthropometrica: A Textbook Of Body Measurement For Sports And Health Courses", University Of New South Wales Press

7. Andreoli A., Scalzo G. et al. (2009) "Body composition assessment by dual-energy X-ray absorptiometry (DXA)", La Radiologia Medica, 2009 114:286-300

8. Cignoni P., Callieri M., Corsini M.et al. "MeshLab: an Open-Source Mesh Processing Tool", Proceedings of Eurographics Italian Chapter Conference 2008: 129-136

9. Lovato C., Castellani U., Giachetti A. (2009) "Automatic Segmentation of Scanned Human Body Using Curve Skeleton Analysis". MIRAGE 2009:34-45 